\title{
Energy harvesting with current sensors to sustain embedded IoT platforms
}

\author{
Matteo Nardello and Davide Brunelli \\ University of Trento, Department of Industrial Engineering, 38122 Trento, Italy \\ \{matteo.nardello, davide.brunelli\}@unitn.it \\ https://www.dii.unitn.it/
}

\begin{abstract}
Powering IoT devices is becoming a major issue, due to the expected number of nodes to be installed in the next years. Powering those devices by harvesting energy from the environment can represent a solution for avoiding battery or $\mathrm{AC} / \mathrm{DC}$ converters. In this paper, we present the study of an electrical energy harvesting power supply using a current transformer sensor, as a feasible solution for powering low power embedded platform without batteries. The proposed system is not dependent on the load, and can be adapted to different scenarios and IoT applications.
\end{abstract}

Keywords: Energy Harvesting, Autonomous systems, Embedded systems

\section{Introduction}

Nowadays energy harvesting systems have emerged as a prominent research area and continues to grow at a rapid pace. Providing solutions for avoiding the need for batteries or power switches, would support the widespread of IoT devices as well as distributed monitoring systems. A wide range of applications suitable to be powered by harvesters are starting to appear [1-5], including distributed wireless sensor nodes for data streaming [6], embedded and implant sensors for wearable applications [7], sensor data acquisition from unmanned vehicles [8], smart merters [9] , and Industrial Internet of Things (IIoT) [10]

For this purpose, we have focused our work on the develop and study of an energy harvesting power supply for low-power embedded systems, in this case, an embedded platform powered by a low-power STM32 MCU and a LoRa radio reffig:block. For tailoring the design to sustain the load, we have started the study from the power requirement of the load and then tailored the main components of the energy harvesting power supply, as the intrinsic characteristics of the used components have an impact on efficiency.

Two different current transformers (CT) sensors have been compared one (CT1) with 1:1000 ratio ${ }^{1}$ and the second one (CT2) with a 1:3000 ratio ${ }^{2}$. Several

\footnotetext{
1 LEM TOP 90-S10/SP2. https://docs-emea.rs-online.com/webdocs/14ce/0900766b814cedb3.pdf

2 Vitec 57PR1673. http://www.viteccorp.com/data/CatalogSensing.pdf
} 
diodes produced by different manufacturers have also been tested, for evaluating the most efficient combination at low currents. Last key component is the energy storage, that should be tailored for sustain the energy requirements of the platform to be powered. Even if, there are innovative technologies available on the market, such as the lithium-ion capacitors [11], supercapacitors are more suitable to achieve an energy-neutral architecture, in our case. The paper is structured as follows: Sections 2 and 3 discuss the selection of the most efficient CT sensor and rectifier components, respectively. Results discussion and sustainability tests are presented in Section 4; then Section 5 concludes this work.

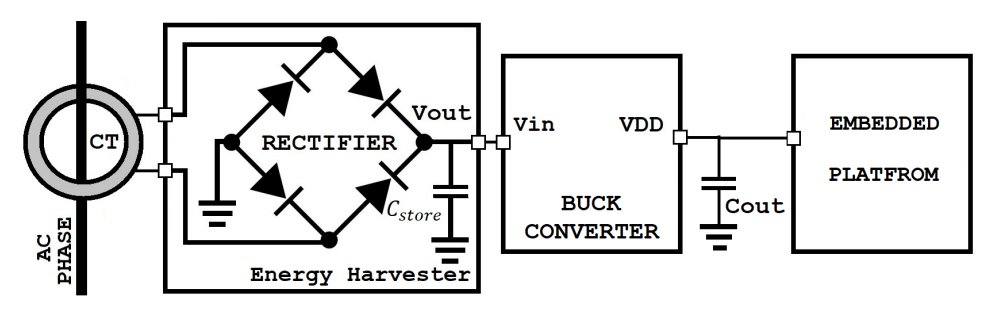

Fig. 1. System architecture block diagram

\section{Rectifier analysis and energy reservoir selection}

Several diodes, with different characteristics, are chosen and analyzed in terms of harvested energy efficiency, to address the best bridge rectifier configuration. Initial tests were conducted using a $100 \mathrm{mF}$ supercapacitor as an energy reservoir and the Vitec CT sensor with a 3:3000 turn ratio. Different primary loads have been simulated using an array made of several incandescent light bulbs and loads, ranging between 100 and 1400 watt. Regarding the rectifiers, bridges with the following diodes have been investigated: SDM10P4 ${ }^{3}, \mathrm{PMEG} 010 \mathrm{ER}^{4}, \mathrm{BAT}^{5}{ }^{5}$, $\mathrm{BAT}_{4} 3^{6}, \mathrm{BAT} 48^{7}, 1 \mathrm{~N} 5711^{8}$ and $1 \mathrm{~N} 4007^{9}$, and results comparison is in Figure $2 \mathrm{a}$. In this respect, the time required for charging the supercapacitor to a specific voltage can vary dramatically, due to the characteristic of the selected diodes (i.e. BAT48 vs IN5711).

\footnotetext{
3 SDM10P45. https://www.mouser.it/datasheet/2/115/ds30287-71260.pdf

4 PMEG2010ER. https://assets.nexperia.com/documents/data-sheet/PMEG2010ER.pdf

5 BAT30. https://www.st.com/resource/en/datasheet/bat30.pdf

6 BAT43. https://www.vishay.com/docs/85660/bat42.pdf

7 BAT48. https://www.st.com/resource/en/datasheet/bat48.pdf

8 1N5711. https://www.st.com/resource/en/datasheet/1n5711.pdf

9 1N4007. https://www.diodes.com/assets/Datasheets/ds28002.pdf
} 


\subsection{Bridge selection}

To evaluate the small differences between the diodes, another set of tests using a smaller supercapacitor have been conducted, this time using only the 3 selected bridges: BAT43, BAT48, and SDM10 since they are the most efficient at lower input current, when the energy that can be harvested is limited. After a performance comparison, BAT48 was selected for further experiments, as the most efficient at lower primary loads. Results are summarized in 2 .

\subsection{Supercapacitor selection}

The energy reservoir, used as the accumulator for the harvested energy, is usually tailored and calculated for the specific application requirements. Starting from the amount of energy consumed by the specific task set, the capacitance of the supercapacitor is chosen accordingly. Three different supercapacitors have been tested. We have used a $5.5 \mathrm{~V} 100 \mathrm{mF}$ supercapacitor, a $4.5 \mathrm{~V} 22 \mathrm{mF}$, and a series of two $12 \mathrm{~V} 90 \mathrm{mF}$. To prevent the power line to drop below the brownout of the platform, another smaller capacitor has been inserted after the buck converter connected to the board power line. This capacitor is used for withstanding the current peak associated with the radio transmission.

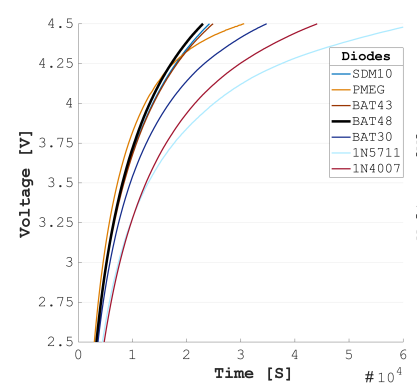

(a)

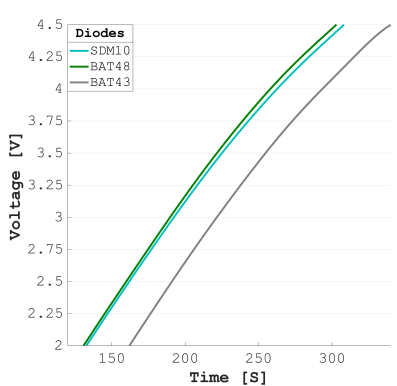

(b)

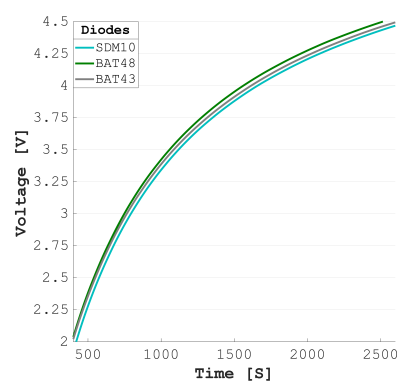

(c)

Fig. 2. a)Charging curves of a $100 \mathrm{mF}$ supercapacitor, using 7 different diodes. For clarity, the figure shows curves ranging from 2.5 to $4.5 \mathrm{~V}$. b/c)Charging curves of a 22 $\mathrm{mF}$ supercapacitor, while harvesting energy from a $500 \mathrm{~W}$ (b) and $1400 \mathrm{~W}$ (c) primary load. For clarity, the figure shows curves ranging from 2 to 4.5 volts

\section{CT sensor analysis}

Like any transformer, a current transformer consists of by a primary winding, a core, and a secondary winding. In our case, the primary is the wire where the CT sensor is clamped on, while the secondary winding is a characteristic of the sensor itself. We have studied two different sensors (CT1) and (CT2) with respectively 1000 and 3000 secondary turns. The relation between the current 
flowing in the primary and the current in the secondary, is described by $3 \mathrm{a}$. Contrarily from the expected the sensor with a lower turning ratio charges the supercapacitor faster, in contrast, with 3a. Experimental tests have highlighted how the output current is affected by the magnetic coupling and impedance mismatch between the CT and the bridge rectifier and that a higher number of primary winding contributes to a better magnetic coupling, thus higher output current in secondary. Result while charging a $100 \mathrm{mF}$ supercapacitor with a 500 $\mathrm{W}$ primary load is presented in Figure $3 \mathrm{~b}$. As it can be noted, the LEM sensor charges the supercapacitor 8 times slower than the Vitec while having a higher turning ratio.

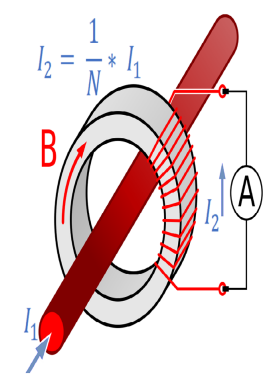

(a)

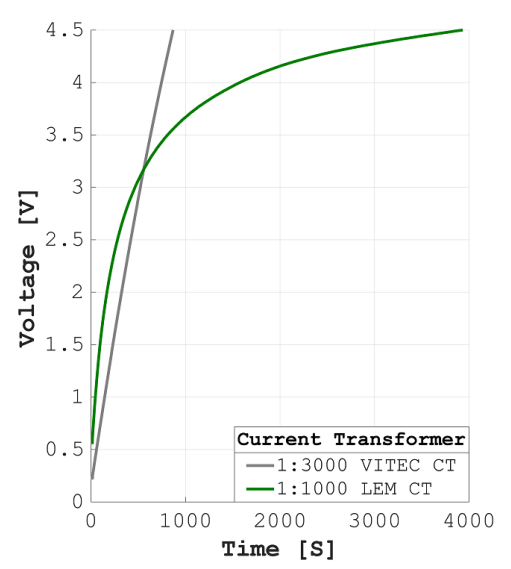

(b)

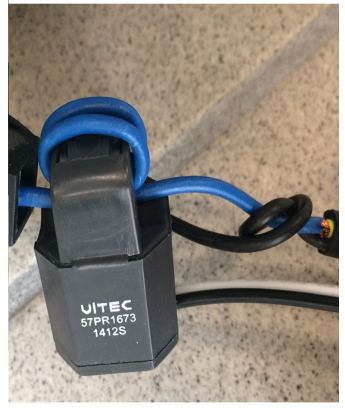

(c)

Fig. 3. a) Working principle of a current transformer. b) Charging curves using two different CT. c) Picture of the installed sensor, with 3 turns of the primary for a ratio of 3 to 3000

\section{Sustainability Analysis}

Once selected the main components of the energy harvesting power supply, we moved our tests to power an embedded platform based on a STM32L4 MCU and an SX1276 LoRa radio. To power the board, an efficient ultra-low-quiescentcurrent buck convert is connected between the high voltage input supercapacitor and the power line of the board. Another smaller capacitor is connected to the output of the buck converter to withstand the peak current associated with the transmission of a LoRa packet. The code running inside the embedded platform simulate a typical scenario where it is required to acquire some data, analyze it and then send using a radio channel:

- Data acquisition: The first task of the board is to acquire a signal using the internal ADC. While in this state, the CPU of the microcontroller is offloaded thanks to the use of the DMA, requiring just $200 \mu \mathrm{A}$.

- Data analysis: Acquired data is first filtered and then analyzed. For simulating a heavy task, the board computes an FFT of the acquired data. In this case, the energy requirement is proportional to the frequency of the MCU. 
- LoRa transmission: Last task is data streaming. Every 5 minutes, a report is sent to a remote server, with all the data collected and analyzed. This is the most demanding task in terms of energy with a current peak of $120 \mathrm{~mA}$.

The first two tasks are repeated every $500 \mathrm{~ms}$, while data is transmitted using the radio every 5 minutes. This is the time for a complete cycle used for evaluating the sustainability of the whole system. This analysis has been carried out by monitoring the voltage of our energy reservoir, that should increase, or at least stay constant, between two successive cycles. This means that the energy harvested is equal to or greater than the energy used by the load. Another range of tests has been conducted to create a relation between the CPU frequency and the minimum primary load for ensuring self-sustained operation, with a packet transmission every 5 minutes. Frequency between 1 and $48 \mathrm{MHz}$ has been tested. Results are presented in Table 1. Figure 4 presents the voltage trend of the input supercapacitor, during the execution of the 3 tasks above. As it can be noted, the node is able to self-sustain its operation, avoiding the need for a battery or an $\mathrm{AC} / \mathrm{DC}$ converter.

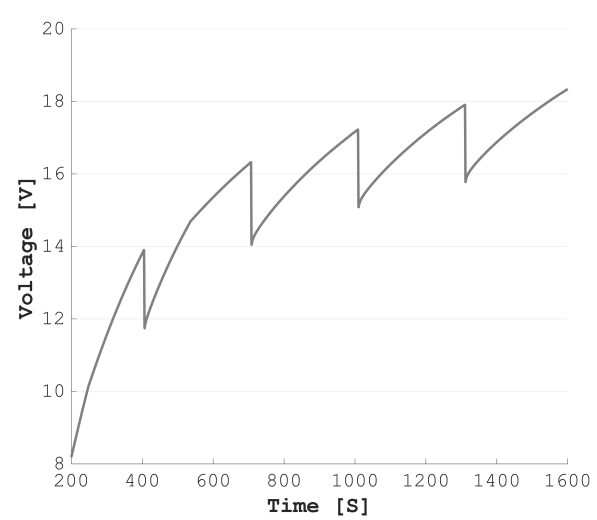

(a)

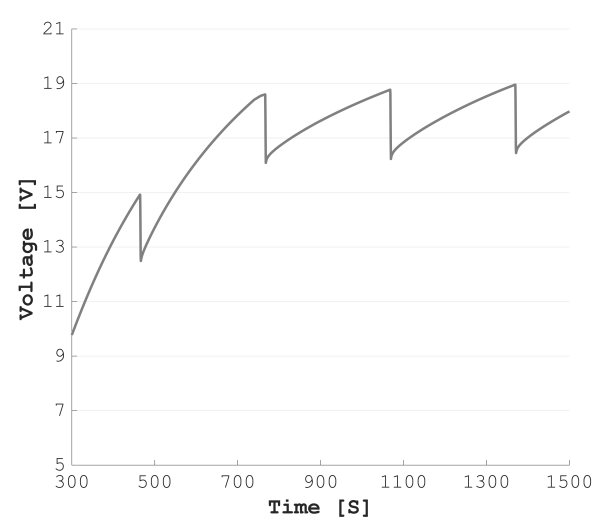

(b)

Fig. 4. Input supercapacitor voltage trend, while powering the embedded platform. a) CPU clocked at $8 \mathrm{MHz}$ while harvesting from $400 \mathrm{~W}$ primary load. b) CPU clocked at $48 \mathrm{MHz}$ while harvesting from $600 \mathrm{~W}$ primary load. As it can be noted, by scaling the frequency, we can adapt the energy demand to the energy provided by the harvester to meet self-sustainable operation.

Table 1. Relation between CPU frequency and primary load, to ensure selfsustainability

\begin{tabular}{l}
\hline Primary load [W] \\
\hline Frequency [MHz]
\end{tabular}




\section{Conclusion}

Providing new solutions for avoiding the use of batteries and AC/DC converters can foster the wide spreading of resource-constrained devices for environmental monitoring. Starting from the power requirements of an embedded system to be powered, in this paper, we tested and sized the energy harvesting power supply. Experimental results have demonstrated how components and parameters optimization is mandatory for maximizing the quantity of energy that can be harvested and thus transferred to the load, and how the proposed system is a feasible solution for powering resource-constrained platforms. Moreover, the proposed power supply is transparent to the load and can be adapted to different scenarios.

\section{References}

1. D. Sartori and D. Brunelli, A smart sensor for precision agriculture powered by microbial fuel cells", in: 2016 IEEE Sensors Applications Symposium (SAS). pp. 1-6 (April 2016)

2. T. Kondo, N. Chiwaki, and S. Sugahara, Design and performance of thin-film $\mu$ teg modules for wearable device applications", in 2017 IEEE Electron Devices Technology and Manufacturing Conference (EDTM), Feb 2017, pp. 201-203.

3. Rossi, M., Rizzon, L., Fait, M., Passerone, R., and Brunelli, D. (2014). Energy neutral wireless sensing for server farms monitoring. IEEE Journal on Emerging and Selected Topics in Circuits and Systems, 4(3), 324334.

4. Bergonzini, C., Brunelli, D., and Benini, L. (2010). Comparison of energy intake prediction algorithms for systems powered by photovoltaic harvesters. Microelectronics Journal, 41(11).

5. Porcarelli, D., Brunelli, D., and Benini, L. (2014). Clamp-and-Forget: A selfsustainable non-invasive wireless sensor node for smart metering applications. Microelectronics Journal, 45(12).

6. Brunelli, D., Maggiorotti, M., Benini, L., Bellifemine, F.L.: Analysis of Audio Streaming Capability of Zigbee Networks. In: Wireless Sensor Networks. EWSN 2008. Lecture Notes in Computer Science, vol 4913. Springer, Berlin, Heidelberg (2008)

7. Brunelli, D., Farella, E., Rocchi, L., Dozza, M., Chiari, L., Benini, L.: Bio-feedback system for rehabilitation based on a wireless body area network. In: Fourth Annual IEEE International Conference on Pervasive Computing and Communications Workshops (PERCOMW'06). pp. 5 pp.-531 (March 2006)

8. Rossi, M., Brunelli, D., Adami, A., Lorenzelli, L., Menna, F., Remondino, F.: Gasdrone: Portable gas sensing system on uavs for gas leakage localization. In: SENSORS, 2014 IEEE. pp. 1431-1434 (Nov 2014)

9. G. Dalpiaz, A. Longo, M. Nardello, R. Passerone and D. Brunelli, "A battery-free non-intrusive power meter for low-cost energy monitoring," 2018 IEEE Industrial Cyber-Physical Systems (ICPS), St. Petersburg, 2018, pp. 653-658.

10. Tessaro, L., Raffaldi, C., Rossi, M., and Brunelli, D., "Lightweight Synchronization Algorithm with Self-Calibration for Industrial LORA Sensor Networks," 2018 Workshop on Metrology for Industry 4.0 and IoT, Brescia, 2018, pp. 259-263.

11. Porcarelli, D., Brunelli, D., Benini, L.: Characterization of lithium-ion capacitors for low-power energy neutral wireless sensor networks. In: 2012 Ninth International Conference on Networked Sensing (INSS). pp. 1-4 (June 2012) 